\title{
Perfil del estudiante de pregrado de la
Facultad de Estudios a Distancia de la
Universidad Militar Nueva Granada
}

Henry Acuña Barrantes ${ }^{2}$

José Eduardo Padilla Beltrán ${ }^{3}$

Felipe Alfredo Riaño Pérez ${ }^{4}$

Recibido: 14-07-2016

Aceptado: 04-1 1-2016

\section{RESUMEN}

El presente estudio busca establecer el perfil de los estudiantes de la Facultad de Estudios a Distancia (Faedis) matriculados en el período 2015II en la universidad, junto con aspectos demográficos, socioeconómicos e institucionales $y$, especialmente, intereses personales en cuanto a estudios de maestría a distancia. Se acudió a la metodología descriptiva mediante una encuesta a una muestra del $39,7 \%$ de la población estudiantil activa en los primeros semestres. Se encontró que los estudiantes encuestados están en el rango de 26 a 45 años principalmente, lo que evidencia una población adulto-joven. Éstos, para solventar la matrícula, a pesar de tener un trabajo, deben acudir a créditos bancarios $(84,1 \%)$. Asimismo, se aprecia que éstos requieren que Faedis ofrezca a corto plazo una maestría en alta gerencia (MBA) $(61,9 \%)$ o en tecnologías de la información y la comunicación (TIC) $(16,3 \%)$, cuyos posgrados se caractericen por un enfoque hacia la investigación y la profundización.

1. Artículo de investigación, relacionado con el proyecto institucional de la FAEDIS titulado "El perfil del estudiante de la Facultad de Estudios a Distancia y su expectativa" con vigencia de 2015.

2. Docente Escuela Española de Negocios Internacionales; Economista-Especialista Alta Gerencia, Universidad Militar Nueva Granada; Máster, MIBE, MIB y doctorando DIB, EENI y Universitat de Girona (España), respectivamente; Integrante Grupo Liderazgo, Universidad Militar Nueva Granada; Clasificado A por Colciencias; Profesional Especializado, Facultad de Estudios a Distancia.

Correo electronico: henry.acuna@unmilitar.edu.co

3. Posdoctorado en Educación, Ciencias Sociales e Interculturalidad, Universidad Santo Tomás; Doctor of Education, Newport University; Magíster, Administración y Supervisión Educativa; Universidad Externado de Colombia; Magíster, Evaluación en Educación, Universidad Santo Tomás; Li ᄀcenciado en Matemáticas, Universidad Pedagógica Nacional; Docente Asociado, Director del Centro de Investigaciones, Facultad de Estudios a Distancia; Líder Grupo de Investigación Pedagogía y Didáctica en Educación Superior (PYDES), Universidad Militar Nueva Granada.

Correo electronico: eduardo.padilla@unimilitar.edu.co

4. Doctor en Educación, Universidad de Guadalajara; Ingeniero Civil, Universidad Militar Nueva Granada; Decano Facultad de Estudios a Distancia-FAEDIS.

Correo electronico: felipe.riano@unimilitar.edu.co 
Palabras clave: perfil del estudiante, educación a distancia, educación superior, pregrados.

\title{
Undergraduates' profile at the Education Distance Faculty, UMNG
}

\begin{abstract}
This study seeks to establish the students' profile at the Education Distance Faculty, enrolled during 2015-II at UMNG, including their demographic, socioeconomic and institutional issues and their own interest particularly upon distance education for master's degrees. A descriptive methodology was applied with a survey to a first-semester students sample, 39.7 per cent of the current student population. Results indicate that students surveyed are largely 26-45 years old, evidencing an adult-young population. Despite they are working, they should take banking loans (84.1 per cent) to afford their tuition. They also require in the short term FAEDIS might offer them robust degrees related to MBA (61.9 per cent) or ICTs (16.3 per cent); all of them focused on research and deepening.
\end{abstract}

Keywords: student profile, distance education, Higher Education, undergraduate programs.

\section{Proposta epistemológica para a sistematização de experiências gerada desde a reflexão sobre a prática}

\section{RESUMO}

No estudo a seguir procura-se estabelecer o perfil dos estudantes da Faculdade de Estudos a Distância da UMNG (Faedis) inscritos no período 2015-II na universidade, junto aos aspetos demográficos, socioeconômicos, e institucionais e, especialmente, interesses pessoais 
no que tem a ver com estudos de mestria a distância. Recorreu-se à metodologia descritiva por meio de uma enquete a uma amostra de $37,7 \%$ da população estudantil ativa nos primeiros semestres. Encontrouse que os estudantes sondados acham-se principalmente entre os 26 e os 45 años, o que evidencia uma população adulto-jovem. Eles, bem que trabalham, para se pagar os estudos, devem acudir a créditos bancários $(84,1 \%)$. Do mesmo jeito, constata-se que eles requerem que Faedis ofereça sem demora uma mestria em alta gerência (MBA) $(61,9 \%)$ ou em tecnologia da informação e a comunicação (TIC) (16,3\%); cujos pós-graus caracterizam-se pela investigação e aprofundamento.

Palavras-chave: perfil do estudante, educação a distância, Educação Superior, universitários.

\section{Introducción}

El estudiante que ingresa a la modalidad a distancia y virtual se circunscribe en el marco de cierta flexibilidad (horario-costo), apertura e innovación, pues cuando ingresan a un programa bajo esta modalidad, encuentran una perspectiva diferente de la generada en la oferta de educación presencial. Con respecto a la dinámica de oferta y demanda de la educación a distancia a nivel nacional, se aprecia cómo la educación superior a distancia tradicional (modalidad que no usa tecnología, pues el contenido se entrega en forma escrita $u$ oral), inicia su real incremento en Colombia a partir de 2004, ya que la educación virtual se visualiza a partir de 2007, tal como lo indican los registros estadísticos oficiales (Alvarado \& Calderón, 2013); el inconveniente es que esta modalidad continúa en aumento y no se tiene información relevante de esta población para conocer de primera mano las necesidades, y mucho menos los requerimientos de estos estudiantes.

Por todo lo anterior, el presente estudio tiene como objetivo establecer el perfil de los estudiantes de pregrado de la Facultad de Estudios a Distancia, matriculados en el período 2015-II, mediante una encuesta que revele los aspectos demográficos, socioeconómicos e institucionales con el fin de responder las preguntas de la investigación: ¿Cuál es perfil de los estudiantes de pregrado de la Faedis? y ¿Qué programas de posgrado les interesa cursar? 


\section{Metodología}

La Facultad de Estudios a Distancia de la Universidad Militar Nueva Granada, entidad pública del orden nacional, plantea la necesidad de conocer el perfil de los estudiantes de pregrado matriculados en la cohorte de 2015-II, con el ánimo de contar con la información que permita establecer aspectos fundamentales, tales como el estrato socioeconómico, el rango de edad, el lugar geográfico donde habita, el tipo de institucionalidad $y$, especialmente, el interés en cursar estudios de posgrado. Al conocer estos aspectos, se resuelven problemas como definir las estrategias pedagógicas y metodológicas a fin de orientar los aprendizajes de los estudiantes según la edad, proponer alternativas en la financiación de las matriculas, orientar sus pautas de divulgación de sus programas y, especialmente, la inclusión en los currículos de situaciones problema de acuerdo a los contextos y regiones donde se encuentren los educandos.

Por lo anterior, se acudió al paradigma interpretativo comprensivo con base en el enfoque cualitativo, con metodología descriptiva, mediante la encuesta como técnica. De una población de 2.921 estudiantes de pregrado, a corte julio de 2015, correspondiente a los alumnos activos e inscritos en modalidad virtual y a distancia en pregrado de los programas de la Facultad de Estudios a Distancia, se tomó una muestra de 1160 estudiantes en forma aleatoria, quienes respondieron satisfactoriamente la encuesta, i.e. 39.7\%. En consecuencia, la obtención y recolección de la información se realizó mediante una muestra probabilística aleatoria simple, mediante un instrumento, anexo a la plataforma de consulta web, aplicado a los alumnos de la Faedis.

\section{Población de la Faedis}

A continuación se presenta el consolidado de estudiantes, tanto institucionales como particulares, que se matricularon entre 2009-I y 2015-I en la Faedis. En razón a esto, el mayor número de matriculados corresponde al programa de Administración de Empresas que durante dieciséis semestres sumaron 6.883 alumnos, lo cual da un promedio de 983 estudiantes por semestre. 
Tabla 1. Matriculados entre 2005-I y 2012-II.

\begin{tabular}{|c|c|c|c|c|c|c|c|}
\hline Programa & 2009-1 & $2010-1$ & 2011-1 & 2012-1 & 2013-1 & 2014-1 & $2015-1\left(^{*}\right)$ \\
\hline $\begin{array}{l}\text { Administración de } \\
\text { Empresas }\end{array}$ & 1016 & 1063 & 1105 & 1180 & 932 & 916 & 671 \\
\hline Contaduría Pública & 795 & 954 & 1103 & 1027 & 692 & 588 & 448 \\
\hline Ingeniería Civil & 442 & 619 & 691 & 747 & 580 & 659 & 650 \\
\hline $\begin{array}{l}\text { Relaciones } \\
\text { Internacionales }\end{array}$ & 608 & 840 & 919 & 937 & 620 & 664 & 524 \\
\hline Ingeniería Industrial & 123 & 284 & 340 & 455 & 304 & 325 & 321 \\
\hline $\begin{array}{l}\text { Administración de la } \\
\text { Seguridad y Salud } \\
\text { Ocupacional - ASSO. }\end{array}$ & & & & & 61 & 225 & 307 \\
\hline
\end{tabular}

Fuente: División de Registro y Control Académico por intranet, 2015-I (matriculados 2.921).

Así, podremos observar que el cupo de estudiantes institucionales oscila entre $35-41 \%$, lo cual es importante tener en cuenta para un análisis de este tipo, cuya finalidad es obtener un óptimo punto de equilibrio económico y académico dentro de la UMNG. Asimismo, en otro estudio (Silva y Espinel, 2006) se menciona que "dentro de las principales características, se evidencia que el $55,17 \%$ de la población es de género femenino y el restante 44,83\%, masculino" (p. 240), y esto guarda relación con los resultados arrojados en el presente estudio, donde la tendencia es un aumento del género femenino.

\section{Resultados a partir de la encuesta interna (modalidad virtual y distancia)}

Todos los resultados se obtienen de las encuestas que se realizaron al grupo de estudio entre julio y octubre de 2015, por ende se obtiene la muestra probabilística de 1.160 estudiantes, de una población total de 2.921 estudiantes distribuidos de la siguiente manera:

- Pregrado (91,5\%)

- Posgrado $(4,7 \%)$

- Diplomado (1,3\%) 


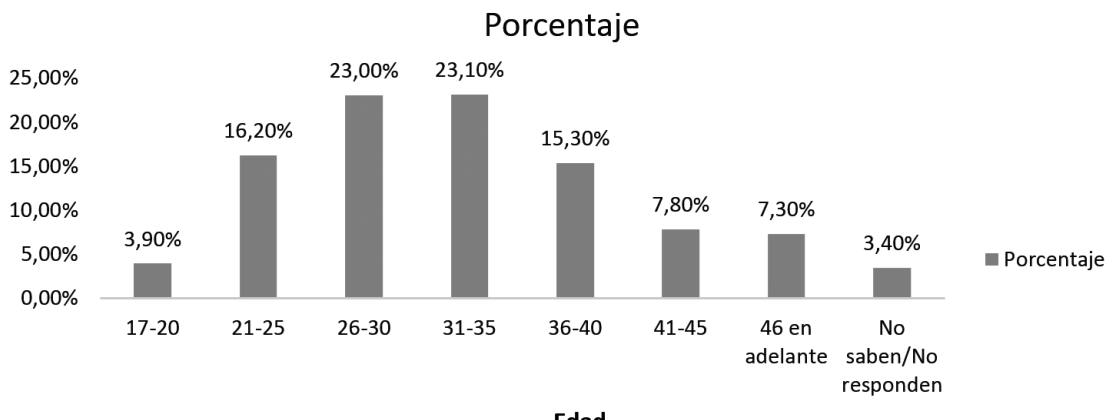

Figura 1. Edad de los estudiantes. Fuente: los autores.

La edad de los estudiantes encuestados, según el criterio de rango, está determinado así: de 17 a 20 años (3,9\%); 21 a $25(16,2 \%) ; 26$ a $30(23,0 \%)$; 31 a $35(23,1 \%) ; 36$ a $40(15,3 \%) ; 41$ a $45(7,8 \%)$; y de 46 años en adelante $(7,3 \%)$. De este modo, se evidencia que la muestra está principalmente en el rango de los 26 a 40 años, con un porcentaje del $61,4 \%$, lo que señala una población adulto-joven, resaltando que un porcentaje importante de educandos son casados o en unión libre $(55,2 \%)$.

Con relación al género, la encuesta arrojó que la población está equilibrada con $41,5 \%$ (femenina) y $58,3 \%$ (masculina).

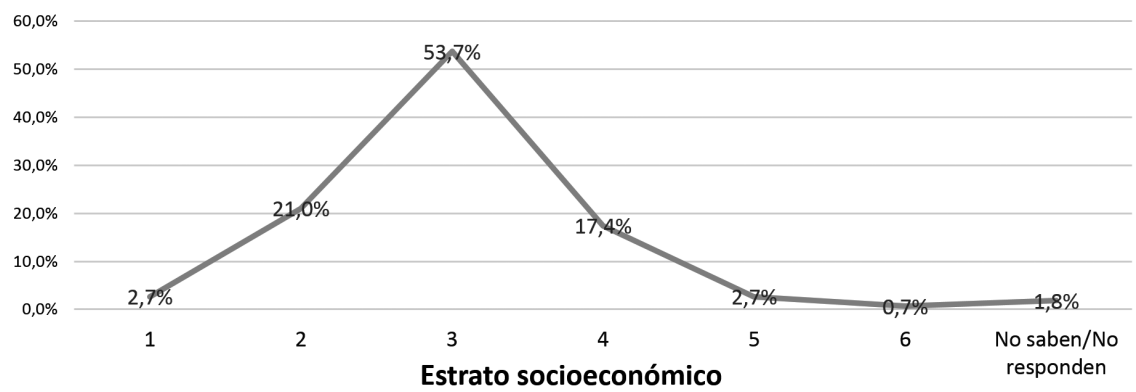

Figura 2. Estrato socioeconómico. Fuente: los autores.

El estrato socioeconómico predominante del estudiante que ingresa a la Faedis es el $3(53,7 \%)$, al que le siguen los estratos 2 y $4(21,0$ y $17,4 \%$, respectivamente. En consecuencia, investigaciones anteriores analizan la situación en la Universidad Militar Nueva Granada, donde se percibe lo siguiente con base en los datos proporcionados por ASCUN, 2000): 
- $1 \%$ de la población pertenece al estrato 1

- En el estrato 2 se ubica 9\%

- En el estrato 3, 52\%

- Al estrato 4 pertenece $24 \%$ de la población estudiantil

- Al 5, 9\%

- Al estrato 6 pertenece $4 \%$.

Con respecto al grado de afinidad con la Universidad, los estudiantes de la Facultad de Estudios a Distancia refieren que se encuentran en la categoría de particulares $(51,8 \%)$ e instituciones $(46,8 \%)$, entendidos los últimos como miembros de las Fuerza Pública, sus cónyuges e hijos menores de 25 años.

\section{Ubicación geográfica}

Referente a la zona en Colombia donde residen los estudiantes encuestados de la facultad, éstos se encuentran principalmente Bogotá $(60,5 \%)$; otras ciudades y municipios de Colombia $(21,6 \%)$ y del mundo (1.2\%).También se evidenció un porcentaje, aunque mínimo, pero cerca al campus, alumnos de la Sabana-Centro $(3,5 \%)$.

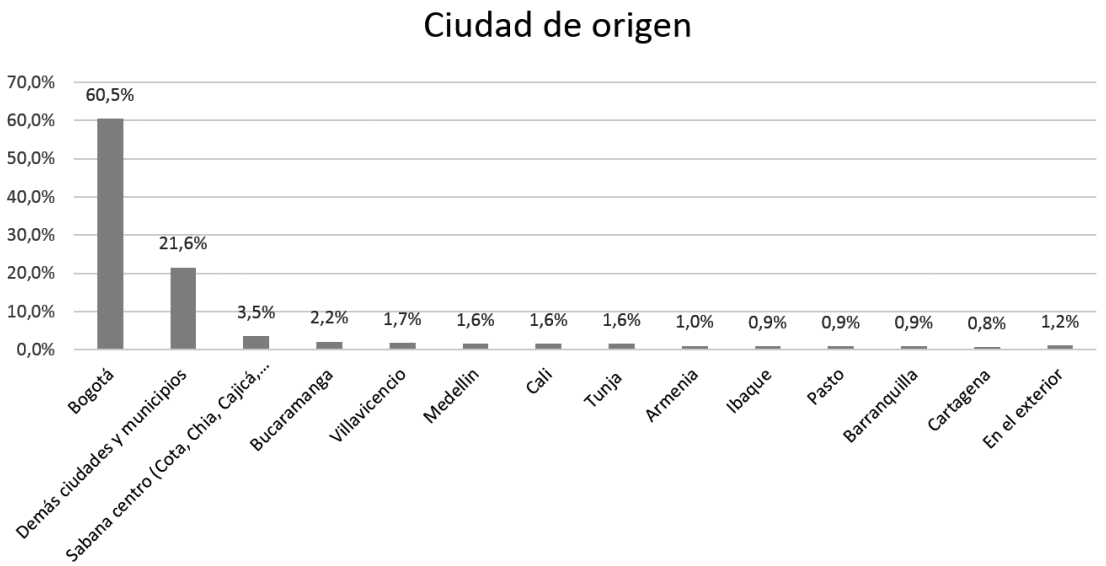

Figura 3. Ubicación geográfica. Fuente: los autores. 


\section{Aspectos económicos}

Los alumnos matriculados en la Faedis en gran medida dependen económicamente de sus propios ingresos, ya que de los 1160 que respondieron la encuesta el $83.9 \%$ trabaja y dependen de su salario para el sostenimiento diario y el pago de matrícula.

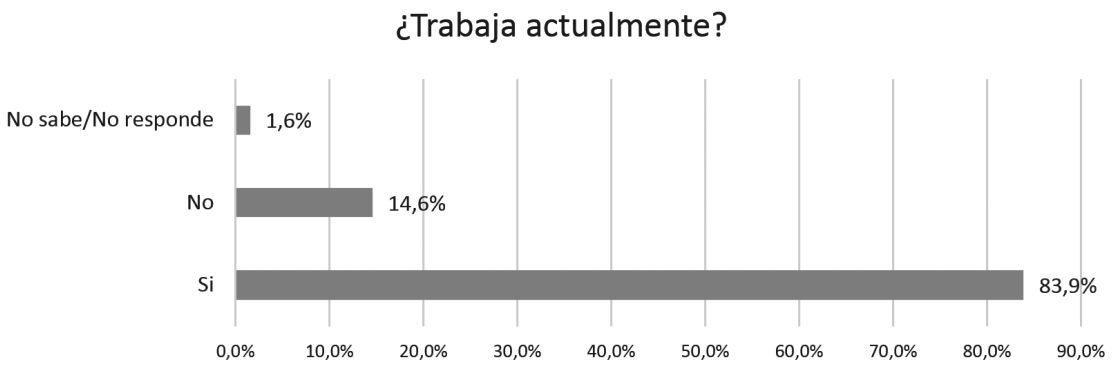

Figura 4. Situación laboral. Fuente: los autores.

En contraste con el estudio presentado por Acuña (2014), en el cual los estudiantes presenciales de la UMNG, dependen de su familia para sobrellevar el costo de manutención y matrícula. En ese mismo estudio, los que trabajan "devengan entre uno y dos salarios mínimos legales mensuales vigentes" (p. 157); mientras que un estudio anterior presentó que los estudiantes "trabajan y la mayoría de éstos devengan entre uno y dos salarios mínimos 84,09\%" (Silva y Espinel; 2006, p. 242).

Por tanto, la mayoría de este grupo de estudiantes que ingresan a un programa a distancia y virtual para pagar la matrícula, a pesar de tener un trabajo, deben acudir a solvencias externas mediante: créditos bancarios $(84,1 \%)$, tarjetas de crédito $(8,4 \%)$, créditos educativos y becas en el exterior (Icetex) $(1,4 \%)$; y sólo lo realizan con recursos propios $(1,1 \%)$; otros estudiantes acuden a su familia en un porcentaje de o, $9 \%$; mientras que $3,9 \%$ aún no tienen certeza de cómo financiar el próximo semestre.

\section{Institucionalidad del estudiante}

Los alumnos encuestados que actualmente se encuentran matriculados en la Faedis, recomendarían a un tercero, la Universidad Militar Nueva 
Granada y a su modalidad a distancia y virtual en $93,8 \%$, lo que representa un modelo de satisfacción del estudiante neogranadino de la Faedis, aunque en prospectiva se pueda tener una totalidad de favorabilidad. Apenas 3,7\% indicó no recomendar el programa que cursa de la Faedis, sin embargo en observaciones los encuestados no indican por qué.

La mayoría de los estudiantes que forman parte de este estudio, seleccionaron a la Facultad de Estudios a Distancia de la UMNG, así:

- Modalidad a distancia y virtual más prestigio institucional (64,9\%)

- Costo matrícula, beneficio y prestigio (24,8\%)

- Influencia familiar (3,2\%)

- Número de semestres por cursar (3,1\%)

- Un porcentaje significativo $(3,1 \%)$ se abstuvo de responder.

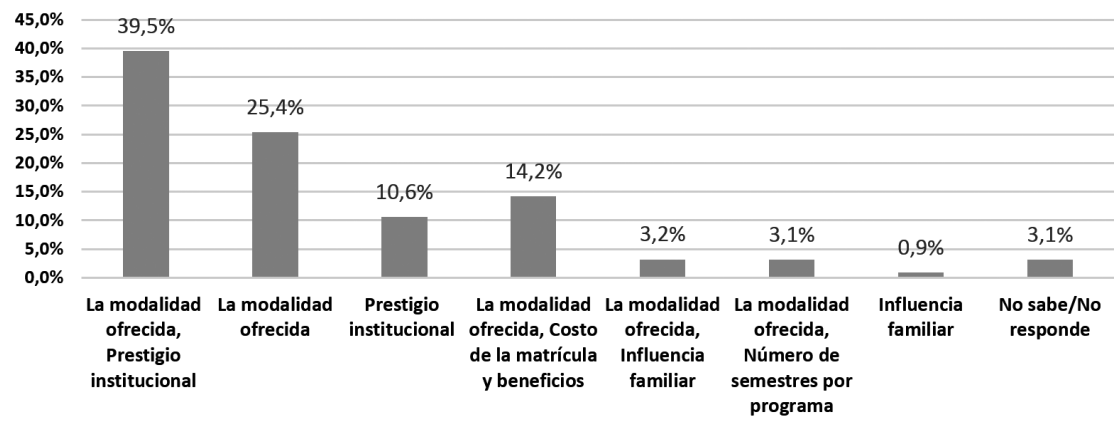

Figura 5. Interés por los programas ofrecidos bajo la modalidad a distancia y virtual. Fuente: los autores.

\section{Percepción de los encuestados sobre el campus}

Algunas preguntas del instrumento estuvieron orientadas a determinar el impacto que tendría el campus Nueva Granada en la comunidad neogranadina que estudia a distancia y está inscrita. Por esta razón, se les preguntó si conocían el Campus Cajicá, y si les quedaría fácil tomar tutorías en esa sede de la Universidad. Como resultado, se obtuvo que de los estudiantes que respondieron estas preguntas, sólo 59,4\% conoce la nueva sede ubicada en Cajicá. Adicionalmente, de acuerdo con la encuesta, se evidenció lo siguiente. 


\section{Cambio de sede de la Facultad de Estudios a Distancia y sus tutorías}

Luego del traslado en el mes de julio de 2015 de la Faedis al Campus Cajicá, existe una población de estudiantes $(67,3 \%)$ que le gustaría continuar recibiendo sus tutorías los sábados en la sede de la calle 100, por el desplazamiento y poco tiempo que tienen en sus trabajos y sólo $19,2 \%$ indicaron que podrían desplazarse a la nueva sede, mientras que $13,4 \%$ indicaron no tener aún una sede específica de interés para recibir su capacitación los sábados.

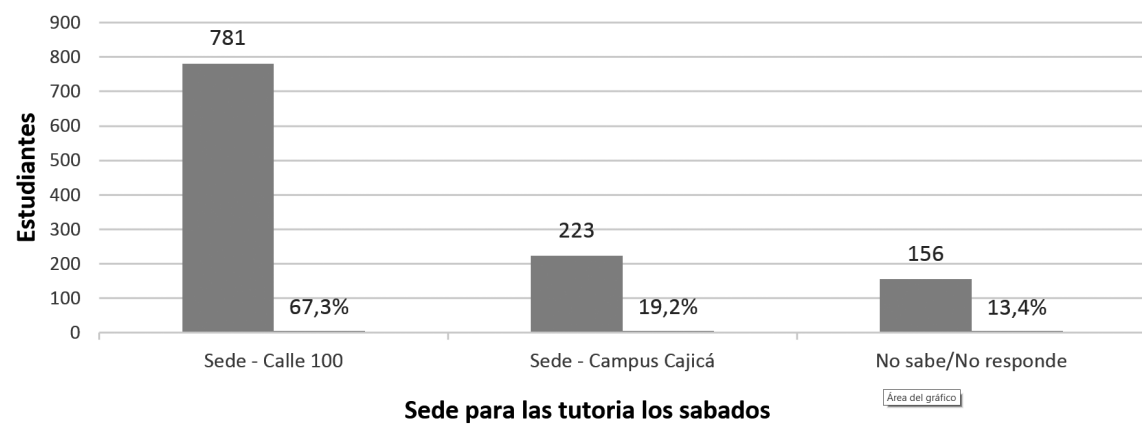

Figura 6. Sedes para recibir las tutorías. Fuente: los autores.

\section{Interés por maestrías a distancia y virtual}

La Facultad de Estudios a Distancia planea a corto plazo ofrecer a sus estudiantes programas de posgrados adicionales existentes (Especialización en Alta Gerencia); por ello, se realizó la pregunta a los encuestados referente al interés a corto plazo de cursar una maestría con la modalidad que ofrece, ante la cual respondieron: Maestría en Alta Gerencia $(61,9 \%)$, TIC $(16,3 \%)$; Relaciones Internacionales $(12,2 \%)$; Educación ( $4,1 \%)$, No sabe/no responde (5,6\%).

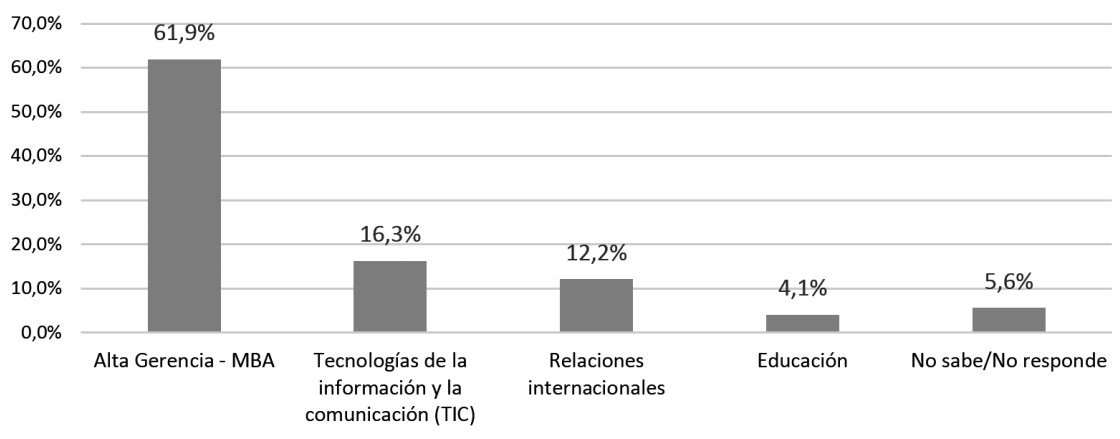

Maestrías de interés

Figura 7. Maestrías demandadas a corto plazo por los estudiantes. Fuente: los autores. 
Y se indagó a su vez qué modalidad en porcentajes (distancia-virtual/ presencial) de dedicación desearían que tuviera la maestría ofrecida para obtener unos porcentajes. Para ello, se les dieron dos opciones de respuesta; una $100 \%$ distancia-virtual, y la segunda distancia-virtual $80 \%$ y presencial $20 \%$. Los resultados son: modalidad a distancia-virtual $100 \%$ $(36,8 \%)$; modalidad distancia-virtual $80 \%$ y $20 \%$ presencial $(53,9 \%)$; y no sabe/no responde $(9,3 \%)$.

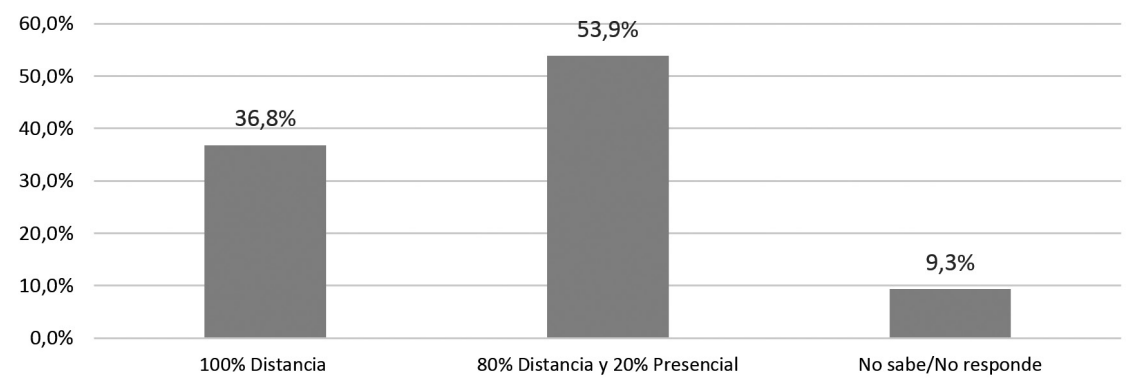

Figura 8. Porcentaje de modalidad a distancia-virtual.Fuente: los autores.

Por último, a los interrogaciones referente a: "Si desean que la maestría esté enfocada hacia la investigación o la profundización", los resultados obtenidos son: investigación $(25,7 \%)$, profundización $(67,6 \%)$, y no sabe/ no responde $(2,2 \%)$. Y en cuanto a que si "Les gustaría que la maestría tuviera sus materias en inglés", $56,2 \%$ les gustaría, y $41,6 \%$ respondió negativamente, mientras que apenas $2,2 \%$ se abstuvo de responder.

\section{Discusiones y conclusiones}

El compromiso interinstitucional de las IES, y específicamente de la UMNG, debe tener rigurosidad en relación con la masiva y poco sistematizada demanda de desarrollar la educación a distancia y virtualidad en todos los escenarios, como una forma de impactar la calidad en la Educación Superior, teniendo en cuenta costos accesibles en aras de reconocer que esta modalidad, tal como afirma Martínez (2013):

[...] No es una opción plena para cualquier persona, y que hace parte de los procesos pedagógicos y de acompañamiento de los programas académicos, identificar las competencias básicas cognitivas y de tecnología y aprendizaje virtual que requieren los 
aspirantes, según la complejidad académica de los programas.

(p. 16)

La educación a distancia en el nuevo entorno virtual, desde diferentes regiones y países, está sujeta a estándares mínimos, en tanto los sistemas de evaluación y acreditación se ajusten a estas modalidades. Sin embargo, las brechas entre las diversas modalidades se van reduciendo e incluso, aunque se aprecia una diferencia del tipo de estudiantes, se visualiza a medida que aumenta la matrícula, una mayor similitud en edades y géneros a las estructuras de los estudiantes presenciales. Igualmente pasa en relación con los docentes, los recursos instruccionales y las dinámicas educativas.

Es importante convocar los programas de la Faedis en Bogotá, pues lo observado en cifras indica la demanda de programa que requiere esta zona central de Colombia, cuyo soporte lo proporcionan los datos estadísticos, donde en la ciudad capital se encuentra la mayor cantidad de población que demanda programas de Educación Superior. Al respecto, el Centro de Centro de Investigación sobre Desarrollo Económico-CEDE (2009) dice lo siguiente:

\begin{abstract}
Aun cuando la oferta de programas de Educación Superior cubre en la actualidad los 32 departamentos del país, persiste la concentración en la matrícula: mientras en Bogotá la cobertura medida en tasa bruta supera el 50\%, en otras diez entidades territoriales no pasa del 10\% del total de la población en edad de cursar estos estudios. El 67,1\% de los estudiantes matriculados en los diferentes programas académicos de pregrado y posgrado en el país se concentran en cinco entidades territoriales: Bogotá, Antioquia, Valle del Cauca, Atlántico y Santander. (p. 23).
\end{abstract}

Referente a la caracterización del estudiante virtual o a distancia, hay estudios que permiten establecer una categorización de la población beneficiaria de los programas de Educación Superior a distancia, que en el caso particular de Colombia, se trata de personas en calidad de bachilleres que adelantan la formación superior o se trata de profesionales interesados en cursar una nueva carrera o adelantar estudios de posgrado. De esta forma, en su gran mayoría, según Arboleda (2007), "[...] el 85\%, son adultos que trabajan y que terminaron su educación media hace más de cinco años, sin que hubieran podido ingresar a la Educación Superior o continuar en ella" (p. 20). 
En la tipificación de esta población, se tiene en cuenta que las tendencias generales observadas en el ámbito internacional, coinciden en términos generales con los rasgos comunes del perfil de estudiantes nacionales matriculados en los programas académicos de esta modalidad, específicamente en lo concerniente a la edad promedio, situación laboral, estado civil, expectativas dentro de su proyecto de vida y estilos de aprendizaje, entre otros factores, tal como se describe a continuación:

- El promedio de edad es 25 años, tendiendo ésta a reducirse, dada la preferencia de la gente joven al manejo de nuevas tecnologías de la información y la comunicación (NTIC).

- Generalmente son educandos que trabajan y buscan profesionalizarse en carreras afines al campo en el cual laboran o al que desean vincularse.

- Desarrollan sus obligaciones académicas en horarios diferentes en relación con su carga laboral y académica (nocturna y fines de semana), inherentes a las múltiples obligaciones académicas y culturales propias de la formación desescolarizada, que tienen además estilos de aprendizaje inclinados hacia la autonomía, autogestión, sentido de responsabilidad en cuanto al aprovechamiento y utilización del tiempo disponible para la jornada académica.

Otro aspecto que se debe analizar a partir de los resultados del instrumento, es lo referente a la nueva sede de la Faedis, y el traslado de los estudiantes para recibir su tutoría; es evidente que la población trabajadora que constituyen un porcentaje importante, se podrían ver afectados al no poder asistir por no otorgamientos de permisos en sus empresas o por otras razones personales. Entonces, resulta de suma importancia desarrollar un estudio para observar la viabilidad de realizar algunas monitorias en la sede calle 100, bien sea entre semana, sábados o domingos, para no entorpecer la educación de estos estudiantes de la Facultad.

Según el DANE, "El estrato 2 corresponde al $36 \%$ de los colombianos, en el estrato 3 está el $31 \%$ de la población del país [...] y el estrato 1 representa el $17 \%$ de la población total" (p. 163). De lo anterior, es preciso tener en cuenta que el impacto socioeconómico de la población atendida se basa, no sólo sobre la persona que recibe sus servicios de formación profesional, sino sobre todo el núcleo familiar y comunidad educativa que incide en la formación del sujeto. 
De igual forma hay que tener en cuenta que existen pocas IES que presentan este tipo de investigaciones según lo examinado desde las indagaciones efectuadas en el presente estudio; sin embargo, se evidencia una serie de datos a partir de los estudiantes de la UMNG en la Faedis, los cuales manifiestan diversas respuestas que son del interés, no solo de la Facultad, sino de todas las unidades académicas que ofrecen estos programas en Colombia. Por consiguiente, se destacan los resultados que arrojó el estudio, los cuales se presentarán según los objetivos planteados en la caracterización de los estudiantes de la Faedis en la UMNG.

A partir de los resultados, por lo menos tres aspectos adquieren importancia; el primero de ellos se relaciona con el estrato socioeconómico de los estudiantes encuestados donde se suman a las evidencias de otros estudios (Ascun, Bogotá, 2000; Bedoya, 2009-2011), los cuales presentan resultados similares sobre este aspecto y reafirman que el estrato socioeconómico predominante es el $3(53,7 \%)$, al que le siguen los estratos 2 y 4 ( $21,0 \%$ y $17,4 \%$, respectivamente). También se tiene que la ciudad donde habitan los estudiantes se encuentran principalmente en Bogotá $(60,5 \%)$, otras ciudades de Colombia $(38.3 \%)$ y del mundo representan un (1,2\%), y un porcentaje de estudiantes de la zona Sabanacentro (Cajicá, Cota, Chía, Calera), cerca al Campus $(3,5 \%)$.

La mayoría de este grupo de estudiantes que ingresan a un programa a distancia y virtual, para poder costear la matrícula, a pesar de contar con un empleo, deben acudir a solvencias externas y de este modo cumplir con el pago de la matrícula semestral, haciéndolo mediante: créditos bancarios $(83,0 \%)$, tarjetas de crédito $(8,4 \%)$, Icetex $(1,4 \%)$; mientras que sólo lo realizan con recursos propios (1,1\%). Asimismo, algunos estudiantes acuden a familiares $(0,9 \%)$; y un porcentaje representativo $(3,9 \%)$ aún no sabe cómo financiarse el próximo semestre.

El segundo aspecto, alusivo a las expectativas de edad de ingreso de los estudiantes encuestados se encuentra en el rango de 26 a 45 años principalmente, con un porcentaje del $(69,2 \%)$, lo que evidencia que se trata de una población adulto-joven. De igual manera, un porcentaje importante son casados y/o en unión libre $(55,2 \%)$. Con relación al género, la encuesta arrojó que la población con $41,5 \%$ es femenina y $58,3 \%$, masculina. Con base en estos datos, de 1.160 alumnos encuestados, la mayoría de los estudiantes que forman parte de este estudio seleccionaron a la Facultad de Estudios a Distancia de la UMNG principalmente por 
modalidad a distancia y virtual y prestigio institucional $(75,5 \%)$, y costo de matrícula y beneficios (14,2\%).

Finalmente, en la investigación se indagó la actitud de los estudiantes, pues cuando inician su actividad académica, observan que la población neogranadina principalmente la componen personas particulares sin ningún vínculo con las Fuerzas Militares ni de otras áreas como la Policía Nacional. Un estudio de mercado realizado en 2011 por Integración. La tienda creativa, indica que:

[...] Se cree que la Universidad es una unidad de formación militar, incluso dependencias gubernamentales, estamentos académicos y congresistas no tienen claro que no hace parte del sistema de defensa, sino que se rige por el Ministerio de Educación. Algunos estudiantes aún preguntan por la instrucción militar. (p. 6)

El estudiante matriculado no tiene ningún vínculo con las Fuerzas Militares ni con la Policía Nacional. Por el contrario, la mayoría de los aspirantes son particulares $(51,8 \%)$, y el estrato socioeconómico de su lugar de residencia, el de la mayoría es 3 . Referente a la pregunta sobre las maestrías, se evidenció que los estudiantes, requieren que la Faedis ofrezca a corto plazo una maestría en esta modalidad, principalmente en Maestría en Alta Gerencia (61,9\%), Tecnologías de la Información y la Comunicación $(16,3 \%)$; y que éstas se caractericen o enfoquen hacia la profundización $(67,6 \%)$.

En la actualidad, el apoyo que la Universidad Militar Nueva Granada otorga a los estudiantes institucionales (UMNG - Resolución o77 de 2013, sobre lineamientos institucionales) con grado de afinidad es muy representativo. Esto se puede evidenciar, y conforme con el presente resultado, en los programas de la Faedis y otras facultades, ya que $46,8 \%$ promedio de los alumnos tiene grado de institucionalidad.

En definitiva, vale subrayar que la creciente oferta de formación profesional a distancia y virtual, está contribuyendo de manera significativa a ampliar la cobertura en la Educación Superior y a superar problemas de marginalidad, exclusión social e inequidad. Además de atender la demanda en los estratos 1, 2 y 3 de las capitales y municipios, a través de esta modalidad se satisfacen necesidades de formación de las zonas urbanas, en ciudades medianas y pequeñas de regiones alejadas 
en las que por lo regular no existen instancias de Educación Superior formal. No obstante, para cumplir este cometido se debe mejorar y complementar la conectividad, tecnología, comunicación e innovaciones pedagógicas en un acuerdo por la apropiación y uso de tecnologías en el campo de la educación.

\section{Referencias bibliográficas}

Acuña, H. (2014). El perfil del estudiante de pregrado de la Facultad de Ciencias Económicas. Revista Educación y Desarrollo Social. 8(2), 146-167.

Alvarado, M. (2013). Diagnóstico Estadístico y Tendencias de la Educación Superior a Distancia en Colombia. En: Arboleda, N. y Rama, C. (eds.). La educación superior a distancia y virtual en Colombia: Nuevas Realidades, pp. 31-46. Bogotá: Virtual Educa.

Arboleda, N. (2007). El perfil socioeconómico de sus egresados en programas de Educación Superior virtual y a distancia. En: Informe final de la Investigación realizada para la Corporación Unificada Nacional de Educación Superior. Bogotá: Corporación Unificada Nacional de Educación Superior.

Arboleda, N. y Rama, C. (eds.). (2013). La educación superior a distancia y virtual en Colombia: Nuevas Realidades. Acesad, Disponible en: http://www.uelbosque.edu.co/ sites/default/files/autoevaluacion_docs/educacion_superior_distancia_virtual.pdf

Asociación Nacional de Universidades-Ascun (2010). Promoción del Desarrollo Humano. En: Caracterización del estudiante universitario de Santa Fe de Bogotá. Bogotá: Ascun.

Bogoya, D. (2013). Elementos de calidad de la Educación Superior en Colombia. Caso de estudio: Universidades. Disponible en: http://www.observatoriovalle.org.co/wpcontent/uploads/2015/01/Estudio-saber-pro.pdf

Brunner, J. \& Uribe, D. (2007). Mercados universitarios. El nuevo escenario de la Educación Superior. Santiago de Chile: Ed. Universidad Diego Portales.

Calero, A. \& Calero, L. (1989). La investigación sobre el estudiante universitario colombiano. Revista Escuela de Administración de Negocios, 7, 53-60.

Centro de Investigación sobre Desarrollo Económico (2009). Informe nacional de: Colombia, Sep. 2009. Bogotá: Cede-Uniandes.

Congreso de la República (1992). Ley 30 de 1992 (art. 53). Por el cual se organiza el servicio público de la Educación Superior. Disponible en: www.mineducacion.gov. co/1621/articles-85860_archivo_pdf.pdf 
DANE (2005). Censo Nacional 2005. Bogotá. Disponible en: https://www.dane.gov.co/ index.php/estadisticas-por-tema/demografia-y-poblacion/censo-general-2005-1

Instituto Colombiano para el Fomento de la Educación Superior-ICFES. (2000). Autonomía universitaria en la selección de estudiantes. Bogotá: Icfes.

Integración. La firma creativa (2011). Estudio de mercado a la Universidad Militar Nueva Granada. Empresa externa de Medellín.

Observatorio Laboral para la Educación (13 de diciembre de 2013). Graduados de Educación Superior por áreas de conocimiento 2001-2012. Disponible en: www. graduadoscolombia.edu.co/html/1732/article-195063.html

OCDE \& BIRF/Banco Mundial (2012). La educación superior en Colombia. Recuperado de: dx.doi.org/10.1787/9789264180710-es

Páramo, G.J. \& Correa C.A. (1999). Deserción estudiantil universitaria. Conceptualización. Revista EAFIT, 35(114), 65-78 Disponible en: http://publicaciones.eafit.edu.co/index. php/revista-universidad-eafit/article/view/1075/967

Silva, A. \& Sarmiento J. (2006). Estudiantes de Economía de la UMNG, ¿cómo son? Revista Facultad de Ciencias Económicas: Investigación y Reflexión, XIV(1), 238-257. Sistema Nacional de Información de la Educación Superior-SNIES. (s.f.). RDisponible en: www.mineducacion.gov.co/\%20Sistema\%20Nacional\%20de\%20Información\%20de\%20 la\%20Educación\%20Superior\%20.\%204\%20instituc.html

Spendolini, M.J. (2005). Benchmarking. Bogotá: Norma.

Universidad Militar Nueva Granada. (2013). Informe enero 31 de 2013. División de Registro y Control Académico. Intranet. Disponible en: 172.16.1.4/index.php?option=com_conte nt\&task=view \&id=44\&ltemid=32 